\title{
Outcomes of a retrospective study on sustained release betamethasone for transient radicular symptoms in patients after Transforaminal Lumbar Interbody Fusion operation
}

Haojie Li

Ningbo University

Yang Wang

Ningbo No 6 Hospital

Weihu Ma (D 806278566@qq.com )

Ningbo No 6 Hospital https://orcid.org/0000-0003-3568-6201

Dingli Xv

Ningbo University

Research article

Keywords: TLIF, Lumbar disc herniation, radicular symptoms, steroid

Posted Date: December 30th, 2019

DOl: https://doi.org/10.21203/rs.2.19710/v1

License: (c) (i) This work is licensed under a Creative Commons Attribution 4.0 International License.

Read Full License 


\section{Abstract}

Objective To observe the efficacy and Complications of gelatin sponge sustained release betamethasone for transient radicular symptoms in patients after TLIF operation.

Method A total of 218 patients with single-level LDH were retrospectively analyzed from March 2017 to July 2019. Patients were divided into observation group and control groups according to different approach.Clinical data such as lasegue sign, VAS score, ODI index were compared, the data analyzed by SPSS 24.0 statistical software.

Results 186 patients were finally included. The observation group has significantly different between the control groups $(\mathrm{P}<0.05)$ at five days after surgery. And there was no significant difference in VAS and ODI between the four groups $(P>0.05)$ at 2-3 months after surgery, . There was no significant difference in blood glucose before and after operation in the observation group $(P>0.05)$, and numeration of leukocyte in the four groups was significantly higher than before operation $(P<0.05)$, but there was no statistically significant difference between the four groups before and after operation $(P>0.05)$. The patients in the four groups had basically the same satisfaction with the final efficacy $(>90 \%)$. In addition, there was the same Complications in four groups(eg.postoperative nausea, vomiting and other complications.)

Conclusion the use of gelatin sponge sustained-release steroids and other mixed solutions can significantly relieve transient radicular symptoms after TLIF operation,and does not influence the occurrence of postoperative complications.

\section{Introduction}

LDH patients have increased by years due to the aging of the population and the wrong lifestyle. TLIF is still a commonly treatment for LDH. But some patients had transient radicular symptoms after surgery, which generally lasted for 2-4 days, and some even more than 10 days. Seriously affected the patient's medical experience and hospital bed turnover efficiency. For such patients, intravenous glucocorticoids and dehydration drugs can significantly relieve symptoms, but there are also clear side effects, including induced aggravation of infection and peptic ulcer,mood disorders,rebound phenomenon and so on[1]. The use of dehydrated drugs can increase the strain on the kidneys. At present, there are studies [2-5] believes that the application of steroids for local epidural blockade of lumbar vertebrae is effective in radiculopathy caused by $\mathrm{LDH}$, It can increase the concentration of local drugs meanwhile reducing the dose of drugs, so as to avoid systemic side effects of drugs.Moreover,the adsorption effect of gelatin sponge, extended the drug release cycles, theoretically can override a postoperative symptoms of radicular, reduce the related side effect.therefore, we hope to use the gelatin sponge adsorb steroid drugs in local place around nerve root in the TLIF operation to alleviate transient radicular symptoms of postoperative early $\mathrm{H}$ However, there are no relevant clinical studies to support this view. we conducted a retrospective randomized, single-blind case-control study to further understand the clinical efficacy and 
complications of the method. Provide theoretical basis and provide clinical data support for further basic research and development of controlled-release formulation.

\section{Methods}

\section{Participation information}

This study was approved by the ethics committee of the hospital, and patients gave informed consent and signed it. A total of 218 patients with LDH who underwent spinal surgery department in Ningbo No.6 Hospital from March 2017 to July 2019 were included. All experimental groups were treated with TLIF. $\mathrm{CT}+3 \mathrm{D}$ reconstruction and MRI examination were performed before operation.

Inclusion criteria:age < 75 years; (2) clinical physical examination and imaging examination confirmed lumbar disc herniation, (3) nerve root symptoms clear, straight leg elevation positive; (4) after conservative treatment no obvious improvement in 3 months, the existence of surgical indications; (5) comply with the principle of informed consent; $\varangle 6 \mathbb{6}$ can accurately express, communication barrier-free.

Exclusion criteria: (1) previous lumbar surgery history; (2) patients with diabetes, active peptic ulcer, malignant tumors, serious liver and kidney dysfunction and diseases of the blood system; (3) acute inflammation, vertebral infection, severe osteoporosis, lumbar congenital deformity and other surgical contraindications.

Preoperative lumbar spine positive lateral position, dynamic position X-ray film, 3D-CT, MRI examination, clear diagnosis, exclusion of root symptoms caused by other factors, followed up for 3 months. There were no significant differences in demographic characteristics and other conditions between the groups $(P>0.05)$, which were comparable (Table 1).

\section{Surgical procedure}

The patient was placed in a prone position with shoulders and bilateral flaps raised. After general anesthesia, routine skin preparation and draping,centered on the body surface projection of the responsible section, cut the skin, subcutaneous soft tissue, muscle, deep fascia by layers, carefully stop bleeding during surgery, place the pedicle screw after expose the vertebral plate and facet joints and fluoroscopy to ensure the position of the screw is correct, the laminectomy is performed, released the adherent nerve root, and the intervertebral cage with the autologous bone is inserted into the intervertebral space after the nucleus pulposus is removed. The gap was filled with gelatin sponge to stop bleeding (Fig 1). After fluoroscopy ensure its position was accurate, the nail rod system was installed and restoration the spondylolysis, and the intertransverse bone graft was performed. The diluted iodine and saline were alternately washed and the incision was placed and drained. The tube was fixed and the observation group placed a gelatin sponge with a mixture of $1 \mathrm{ml}$ betamethasone injection $+2 \mathrm{ml} 2 \%$ lidocaine on the nerve root of the spinal cord ( Fig. 2). In the control group 1, $1 \mathrm{ml}$ betamethasone injection $+2 \mathrm{ml} 2 \%$ 
lidocaine was placed, and in the control group 2, a gelatin sponge with $3 \mathrm{ml}$ normal saline was placed around the spinal nerve roots after decompression, and the control group 3 not do anything. Finally, vancomycin was applied to the wound, and the incision was closed by layers suture, and the drainage tube was opened.

\section{Postoperative treatment}

Four groups of patients were given oxygen routinely, ECG(Electrocardiogram) monitoring, and skin oxygen monitoring for 12 hours. attention was paid to the movement and sensation of legs, and wound drainage, etc. Removed drainage tube when the daily drainage volume was less than $50 \mathrm{ml}$. The patients who wear a lumbar support can get out of bed when the pain was significantly reduced. Postoperative blood reexamination of blood routine, biochemical, timely review of CT(Computed Tomography) or MRI(Magnetic Resonance Imaging) after extubation, regular follow-up, to avoid large waist activity within 3 months.

\section{Measurements}

Before operation and 3 months after operation, Lasegue sign, numeration of leukocyte and blood glucose of the four groups before and after the operation were recorded, including the postoperative hospital stay, wound healing and infection. Clinical efficacy was evaluated by using the modified Macnab criteria at discharge. VAS and ODI were used to assess the pain and functional recovery of the four groups before and after surgery, 1-5 days, 1 month, 2 months, and 3 months after surgery.

\section{Statistical methods}

SPSS 24.0 statistical software was used for data analysis. The measurement data were expressed as mean \pm standard deviation $(x \pm s)$. The comparison between groups was performed by single factor ANOVA test or independent sample t test. The count data were compared by $\chi 2$ test, expressed as $P<0.05$. The difference was statistically significant.

\section{Outcomes}

In the study, 186 patients were finally included after excluding 32 patients. All patients had good intraoperative screws and cages, and no blood transfusion was found. No cases were found in which the screws were placed in the spinal canal and the cage was loose. Among them, the observation group included 46 cases, control group 1:53 cases, control group 2:45 cases, and control group 3:42 cases. The average age of the observation group was $55.6 \pm 7.2 \mathrm{y}$, the average disease duration was $7.3 \pm 4.0 \mathrm{~m}$, the 
prominent segment L3/4:6cases, L4/5:26cases, L5/S1 :14cases, the average operation time was $118 \pm 13.0 \mathrm{~min}$, the average blood loss was $156 \pm 34.5 \mathrm{ml}$, the average preoperative blood glucose was $5.9 \pm .69 \mathrm{mmol} / \mathrm{L}$, and the average preoperative numeration of leukocyte was $6.6 \pm .92 * 10^{9} / \mathrm{L}$. As shown in table 2 and 3, VAS score of postoperative legs pain after the extubation in the observation group was significantly lower than the control groups, and rebound pain was lower than the control groups. The recovery of Lasegue sign as also better than the control groups $(P<0.05)$. The average of postoperative hospital stay was $5.7 \pm 1.4$ days, which was significantly shorter than the control groups. At 2-3 months after surgery, there was no significant difference in VAS and ODI between the four groups $(P>0.05)$. There was no statistically significant difference in blood glucose before and after operation in the observation group $(P>0.05$ ) (table 3 ), numeration of leukocyte postoperative was significantly higher than preoperative in each group $(P<0.05)$, but there was no statistically significant difference between groups $(P>0.05)$ (table 1). The patients in the four groups had the similar satisfaction with the final efficacy (> 90\%) (table 4). In addition, 7cases(15\%), 7cases (13\%), 8cases(17\%) and 6cases (14\%) in the observation group and control group $1 \otimes 2 \varangle 3$ had postoperative nausea, vomiting, leakage of cerebrospinal fluid and other complications (table $5 \rrbracket$.

\section{Discuss}

The mechanisms of symptoms

Although TLIF surgery's curative effect is exact, but patients always complain that pain relief is not complete. After removed drainage tube, the pain worse, Some patients appear even "rebounding pain". By review the literature [2,7-11], we found that the possible mechanisms of transient radicular symptoms after TLIF was as fellows:1.Long-term pressure inflammatory stimulation caused adhesion and edema around nerve roots, which reduced its tolerance to mechanical effects, increased the sensitivity of pain, and produced sensitization of central pain sensation;2.The intraoperative dural membrane floating increases the tension of nerve roots, aggravating the intraoperative strain injury and causing postoperative rebound pain;3.Aseptic inflammation occurs in damaged tissues cause a large number of inflammatory mediators include phospholipase A2, arachidonic acid and its metabolites TNF-2, IL-2, prostaglandins,etc., which imbalance the pro-inflammatory and anti-inflammatory factors.In addition, due to less blood supply around the disc and nerve roots, the clearance of inflammatory factors is slow, so that postoperative pain relief is not complete. 4. Blood oozed inside the incision after extubation, which caused postoperative hematoma to become organized and scar hyperplasia, and increased the stimulation and pulling of nerve roots; 5.The spinal cord and nerve roots are in a state of long-term compression and ischemia, reperfusion injury caused by the release of compression causes postoperative nerve root pain.

Steroid hormones have strong anti-inflammatory effects, reduce inflammatory exudation, and improve local microcirculation, reduce tissue edema, directly act on nerve roots and surrounding tissues, inhibit hyperplasia of nerve roots and surrounding connective tissue, thereby reducing accumulation of local acidic substances and desensitization.[12]lt is widely used for epidural steroid injection (ESI) treatment, 
However, there are risks such as increased infection, cardiovascular failure, glucose metabolism disorder[13,14].the use on clinical is still controversial [2,15].Although the standard operation of injection can reduce the occurrence of complications, but the kambin triangle is still unable to avoid spinal cord vascular injury compared with the nerve. In addition, granule steroids strayed into the blood vessels cause complications such as spinal cord infarction and paraplegia $[4,16]$. Although clinical treatment of postoperative lower extremity root symptoms can be achieved by oral, intravenous steroids, mannitol, etc., it takes a certain dose and time to achieve effective concentration locally,lead to the increase of complications [17], the existing contraindication of patients, such as liver and kidney dysfunction, femoral head necrosis, diabetes, etc.) will increase viscera burden lead to failure. So we used betamethasone, which has a relatively small molecular weight, and we did a statistical analysis of the possible adverse reactions.

\section{Drug selection}

Some literatures[3,5] have pointed out that local anesthetics combined with steroids have better efficacy than local anesthetics alone. In this study, lidocaine belongs to amide local anesthetics, which can continuously relieve neuralgia after local anesthesia block [18], while steroids can reduce the permeability of nerve root vessels of spinal cord and prolong the action time of local anesthetics. In addition, the efficacy of $8 \mathrm{ml}$ volume for epidural block with the same dose of steroids was significantly better than $4 \mathrm{ml}$ [19], but the optimal volume and drug concentration were not reported yet.Considering that the oozing blood of the surgical wound has a certain expansion effect on the drug, we have not expanded the volume of the mixed solution, and adopted a gelatin sponge with better histocompatibility as the carrier of the above-mentioned drug, and using its adsorption function of slow-release function, and because of its soft, won't cause secondary to nerve compression.In order to eliminate the difference between groups, all the four groups were operated by the same operator.Nerve monitoring was used throughout the operation to determine whether postoperative lower limb dysfunction was caused by local anesthetic or intraoperative nerve injury.By observing Figure 3, we found that the VAS scores of legs'pain in the four groups increased on the fourth day postoperation, reaching a peak in 4-5 days, suggesting that the extubation after operation, intraoperative nerve root traction led to increased edema, hematoma compression The same stimulus appeared, but the curve of the observation group became more stable, which enabled the patient to get out of bed earlier and increase functional rehabilitation. From Fig $4 \llbracket 5$, we found that the short-term efficacy of this method is exact, but has no significant effect on the mid-term efficacy. From Fig5, we found that the observation group had the most obvious decrease in ODI index during hospitalization, while the control group mainly focused on postoperative Within 1 month, the patient's earlier bed-out activity was positively correlated with functional recovery, indicating the importance of early pain relief. In addition, the hospitalization time, efficacy satisfaction, and ODI index (within 5 days) of the observation group were superior to the control group, further indicating that the method has greater advantages in patient satisfaction and bed turnover. The literature reported that the complications of topical application of steroids (including the risk of hypotension, spinal cord infarction, 
meningitis) compared with the control groups, were not found in our study. Incision healing, infection rate, and blood glucose changes were not significantly different from the control groups, as reported by Bogduk [20], further confirming the safety of the method. Although Numeration of leukocyte of patients before and after surgery were significantly different, no differences were found between the groups. We considered that the increase in numeration of leukocyte count after surgery was a stress reaction caused by intraoperative trauma, and was not related to the use of steroids [21-23]. In the observation group, 7 patients had a postoperative nausea and vomiting rate of $15 \%$, which was not significantly increased compared with the control groups, and was considered to be related to the use of intraoperative anesthetics. The control group 1 directly infiltrated the mixed solution, and the drug was easily lost under the suction of the negative pressure drainage tube, and could not play a role locally. Therefore, the role of the gelatin sponge could not be ignored. Some patients have a relatively long course of disease, high preoperative inflammation index, and there may be hyperalgesia. Secondly, hemostasis does not completely dilute the drug during the operation, resulting in serious drug loss. In addition, if the patient expects higher efficacy, VAS score will increase, we recommend strict hemostasis during surgery, appropriate delay of negative pressure drainage release time.

\section{Matters needed attention}

The dose of the drug in this study was determined based on the existing literature, not sure the minimum effective dose, the optimal drug ratio, and the effective time of the drug. In addition,the study object in this paper is single-center case and the sample size is small.

\section{Conclusion}

Through this study, the use of gelatin sponge sustained-release steroids and $2 \%$ lidocaine mixed solutions can significantly improve transient radicular symptoms after TLIF, shorten hospitalization time, help patients get out of bed early, improve clinical satisfaction, the effect is exact, and local small dose, It is safe and feasible to apply steroids in a short period of time, and the placement of gelatin sponge will not cause secondary compression. Has the advantages as fellow: 1. Avoid the occurrence of similar puncture accidental complications 2 . Instantly reach a certain concentration in the local, avoid systemic administration 3. Have a certain sustained release effect 4. Reduce the dilution and loss of the drug caused by drainage and oozing. Simple operation in direct vision environment 6 . Make patients get out of bed early and accelerate recovery.

\section{Declarations}

Acknowledgements

1. Ethics approval and consent to participate:This study was approved by the ethics committee of the Ningbo No. 6 hospital, and patients gave informed consent and signed it! 
2. Consent for publication:This manuscript is approved by all authors for publication.

3. Availability of data and materials:The data and material are consented by patients to use in this clinical study.

4. Competing interests:No conflict of interest exits in the submission of this manuscript

5. Funding:We have no funding in this research.

\section{Authors' contributions:}

Ma weihu:conceived and designed the study,and is the Surgeon of all operation

Wang Yang and Xu Dingli :performed the experiments

Ma weihu and Li haojie: data analysis and write manuscript,reviewed and edited the manuscript

7.Acknowledgements:We would like to thank all of the patients who participated in this study.

\section{Abbreviation}

\begin{tabular}{|c|c|}
\hline TLIF & Transforaminal Lumbar Interbody Fusion \\
\hline LDH & Lumbar Disc Herniation \\
\hline VAS & Visual Analogue Scale \\
\hline ODI & Oswesry Disability Index \\
\hline
\end{tabular}

\section{References}

[1]H G, W F, M T, A P, L A, L H, W S, R C, A M, LA W, E C, AL A (2015) Oral steroids for acute radiculopathy due to a herniated lumbar disk: a randomized clinical trial. JAMA 313 (19):1915-1923

[2] DR, F., SM, W., AJ, P., \& H, B. (2019). Are Epidural Steroid Injections Beneficial for Patients With Degenerative Lumbar Spondylolisthesis? Clinical spine surgery, 32(8), 309-312.

[3]L, M., KA, C., V, P., BW, W., \& Y, M. (2012). Management of chronic pain of cervical disc herniation and radiculitis with fluoroscopic cervical interlaminar epidural injections. International journal of medical sciences, 9(6), 424-434.

[4]D, L., S, H., \& S, C. (2016). The Incidence of Intradiscal, Intrathecal, and Intravascular Flow During the Performance of Retrodiscal (Infraneural) Approach for Lumbar Transforaminal Epidural Steroid 
Injections. Pain medicine (Malden, Mass.), 17(8), 1416-1422.

[5] CE, R. (2018). Lumbar Epidural Steroid Injections. Physical medicine and rehabilitation clinics of North America, 29(1), 73-92.

[6]PG C, GY J, YS Y, DA S (2019) Clinical Effectiveness of Percutaneous Epidural Neuroplasty According to the Type of Single-Level Lumbar Disc Herniation : A 12-Month Follow-Up Study. Journal of Korean Neurosurgical Society 62 (6):681-690

[7]J H, S X, Z W, Z Y (2016) Microendoscopic discectomy versus open discectomy for lumbar disc herniation: a meta-analysis. European spine journal : official publication of the European Spine Society, the European Spinal Deformity Society, and the European Section of the Cervical Spine Research Society 25 (5):1373-1381

[8]A D, MA D, A G (2019) Pathologic Basis of Lumbar Radicular Pain. World neurosurgery 128 (undefined):114-121

[9] M U, K Ç, M S (2017) The surgery and early postoperative radicular pain in cases with multifocal lumbar disc herniation. Medicine 96 (9):e6238

[10]K W, JP B, S Y, X H, L L, XH X, XT W (2016) A cohort study comparing the serum levels of pro- or antiinflammatory cytokines in patients with lumbar radicular pain and healthy subjects. European spine journal : official publication of the European Spine Society, the European Spinal Deformity Society, and the European Section of the Cervical Spine Research Society 25 (5):1428-1434

[11]J H, M Y, S O, K S, Y I, K T (2006) Relationship between low-back pain, muscle spasm and pressure pain thresholds in patients with lumbar disc herniation. European spine journal : official publication of the European Spine Society, the European Spinal Deformity Society, and the European Section of the Cervical Spine Research Society 15 (1):41-47 
[12]L M, V S, KA C, V P, KS D, MV B (2012) Effect of fluoroscopically guided caudal epidural steroid or local anesthetic injections in the treatment of lumbar disc herniation and radiculitis: a randomized, controlled, double blind trial with a two-year follow-up. Pain physician 15 (4):273-286

[13]Multistate outbreak of fungal infection associated with injection of methylprednisolone acetate solution from a single compounding pharmacy - United States, 2012 (2012). MMWR Morbidity and mortality weekly report 61 (41):839-842

[14]R C, R H, J F, R F, C B, T D, SD S, J J (2015) Epidural Corticosteroid Injections for Radiculopathy and Spinal Stenosis: A Systematic Review and Meta-analysis. Annals of internal medicine 163 (5):373-381

[15]S K, K T, T Y, K A, T M, A G, H B (2010) Pathomechanisms of sciatica in lumbar disc herniation: effect of periradicular adhesive tissue on electrophysiological values by an intraoperative straight leg raising test. Spine 35 (22):2004-2014

[16]RV S (2014) Paraplegia following thoracic and lumbar transforaminal epidural steroid injections: how relevant are particulate steroids? Pain practice : the official journal of World Institute of Pain 14 (4):297300

[17]NA M, MD M, EP A (2013) Transforaminal epidural steroid injections prevent the need for surgery in patients with sciatica secondary to lumbar disc herniation: a retrospective case series. Canadian journal of surgery Journal canadien de chirurgie 56 (2):89-96

[18]S A, U L, BA M, C M (1990) Prolonged relief of neuralgia after regional anesthetic blocks. A call for further experimental and systematic clinical studies. Pain 43 (3):287-297

[19]EH C, HS P (2015) Effect of High-Volume Injectate in Lumbar Transforaminal Epidural Steroid Injections: A Randomized, Active Control Trial. Pain physician 18 (6):519-525 
[20]B N. Lumbar transforaminal access. In: Bogduk N, editor. Practice guidelines for spinal diagnostic and treatment procedures. San Francisco (CA): International Spine Intervention Society; 2013. p. 465.

[21]S H, SJ J, KB W, TD C, JH S, CM B, LG J (2016) Is There an Association of Epidural Corticosteroid Injection With Postoperative Surgical Site Infection After Surgery for Lumbar Degenerative Spine Disease? Spine 41 (19):1542-1547

[22]JM B, B LG, Y M (2013) Side effects of corticosteroid injections: what's new? Joint, bone, spine : revue du rhumatisme 80 (4):363-367

[23]JG S, GC B, T S, M H, DE G, SC W (2017) The effect of preoperative lumbar epidural corticosteroid injection on postoperative infection rate in patients undergoing single-level lumbar decompression. The spine journal : official journal of the North American Spine Society 17 (9):1209-1214

\section{Tables}

Table 1 Detail baseline characteristics 


\begin{tabular}{|c|c|c|c|c|c|}
\hline Parameter & $\begin{array}{l}\text { Observation } \\
\text { group }\end{array}$ & $\begin{array}{l}\text { Control } \\
\text { group } 1\end{array}$ & $\begin{array}{l}\text { Control } \\
\text { group } 2\end{array}$ & $\begin{array}{l}\text { Control } \\
\text { group } 3\end{array}$ & $\begin{array}{c}\mathrm{P} \\
\text { value }\end{array}$ \\
\hline No.of patients(n) & 46 & 53 & 45 & 42 & \\
\hline Age(year) & $56.0 \pm 7.6$ & $54.8 \pm 7.1$ & $53.6 \pm 6.4$ & $58.3 \pm 6.2$ & .013 \\
\hline Male/Females(n) & $32 / 14$ & $33 / 20$ & $29 / 16$ & $23 / 19$ & .547 \\
\hline Symptom duration $\square$ months $\square$ & $8.6 \pm 3.9$ & $7.8 \pm 2.8$ & $7.7 \pm 2.7$ & $8.1 \pm 3.0$ & .443 \\
\hline \multicolumn{6}{|c|}{ Herniation level(n) } \\
\hline L3/L4 & 6 & 5 & 3 & 3 & \multirow[t]{3}{*}{.413} \\
\hline L4/L5 & 26 & 30 & 22 & 17 & \\
\hline L5/S1 & 14 & 18 & 20 & 22 & \\
\hline Surgical time (min) & $119 \pm 11.2$ & $115 \pm 9.9$ & $115 \pm 8.1$ & $120 \pm 12.5$ & .018 \\
\hline Blood loss (mla & $142 \pm 33$ & $134 \pm 28$ & $138 \pm 22$ & $147 \pm 37$ & .220 \\
\hline $\begin{array}{l}\text { Half/Complete } \\
\text { laminectomy(n) }\end{array}$ & $35 / 11$ & $44 / 9$ & $39 / 6$ & $31 / 11$ & .397 \\
\hline NOL』*109/L口 & $6.86 \pm 1.5$ & $7.2 \pm 1.4$ & $7.4 \pm 1.5$ & $6.5 \pm 1.2$ & .021 \\
\hline $\mathrm{PBG} \square \mathrm{mmol} / \mathrm{L} \square$ & $5.8 \pm .79$ & $5.7 \pm .73$ & $5.6 \pm .73$ & $6.1 \pm .67$ & .021 \\
\hline Stay post operation (day) & $5.3 \pm 2.0$ & $6.1 \pm 2.0$ & $6.3 \pm 1.6$ & $6.8 \pm 1.4$ & .002 \\
\hline Lasegue sign $\square^{\circ} \square$ & $44.8 \pm 8.8$ & $43.1 \pm 7.1$ & $46.9 \pm 9.3$ & $46.8 \pm 5.6$ & .059 \\
\hline
\end{tabular}

NOL:Numeration of leukocyte PBG:Postprandial blood glucose

Table 2 VAS score of legs pain from preoperative to postoperative 
Parameter Observation Control group 1 Control group 2 Control group 3P value group

\begin{tabular}{|cccccc|}
\hline Pre-operation & $6.4 \pm 1.5$ & $6.6 \pm 1.1$ & $6.7 \pm 1.4$ & $6.4 \pm 1.1$ & .320 \\
\hline Day 1 & $2.3 \pm .8$ & $2.4 \pm .7$ & $2.3 \pm .7$ & $2.1 \pm .5$ & .225 \\
\hline Day 2 & $1.9 \pm .8$ & $2.2 \pm .6$ & $2.0 \pm .7$ & $1.9 \pm 0.6$ & .221 \\
\hline Day 3 & $2.1 \pm .7$ & $2.4 \pm .7$ & $2.3 \pm .7$ & $2.3 \pm .7$ & .254 \\
\hline Day 4 & $2.3 \pm 1.2$ & $2.7 \pm .7$ & $2.6 \pm .6$ & $2.5 \pm .6$ & .129 \\
\hline Day 5 & $2.1 \pm 1.0$ & $2.7 \pm 1.0$ & $2.8 \pm .8$ & $2.7 \pm .7$ & .001 \\
\hline Month 1 & $1.3 \pm 0.6$ & $1.7 \pm 0.7$ & $1.4 \pm 0.5$ & $1.6 \pm 0.5$ & .033 \\
\hline Month 2 & $.8 \pm 0.4$ & $1.0 \pm 0.5$ & $.9 \pm 0.4$ & $.9 \pm 0.5$ & .316 \\
\hline Month 3 & $.7 \pm .5$ & $0.7 \pm 0.4$ & $0.6 \pm 0.4$ & $0.6 \pm 0.4$ & .799 \\
\hline
\end{tabular}

Day 1:postoperative 1 day;Month 1:postoperative 1 month

Table 3 The D-value between preoperative and postoperative 3 days

\begin{tabular}{|c|c|c|c|c|c|}
\hline \multicolumn{6}{|c|}{ The D-value between Preoperative and Postoperative 3 days } \\
\hline Parameter & $\begin{array}{l}\text { Observation } \\
\text { group }\end{array}$ & Control group 1 & Control group 2 & Control group 3 & $P$ value \\
\hline Lasegue sign $\square^{\circ}[$ & $13.61 \pm 4.8$ & $10.5 \pm 5.7$ & $8.4 \pm 4.2$ & $8.5 \pm 8.9$ & .000 \\
\hline PBG $\square \mathrm{mmol} / \mathrm{L} \square$ & $-.20 \pm .9$ & $-.18 \pm .8$ & $.04 \pm 1.0$ & $-.12 \pm .5$ & .486 \\
\hline NOL ${ }^{*} 109 / \mathrm{L} \square$ & $3.1 \pm 1.6$ & $2.8 \pm 1.7$ & $2.9 \pm 1.4$ & $3.6 \pm 2.5$ & .201 \\
\hline
\end{tabular}

Table 4 Effective standard postoperative 3 months

\begin{tabular}{|c|c|c|c|c|}
\hline \multicolumn{5}{|c|}{ Effective standard in four groups\n] } \\
\hline Parameter & $\begin{array}{l}\text { Observation } \\
\text { group }\end{array}$ & Control group 1 & Control group 2 & 2 Control group 3 \\
\hline excellent & 13 & 15 & 11 & 10 \\
\hline good & 29 & 33 & 31 & 28 \\
\hline better & 2 & 4 & 3 & 2 \\
\hline poor & 2 & 1 & 0 & 2 \\
\hline Rate of excellent and good & $91.3 \%$ & $90.5 \%$ & $93.3 \%$ & $90.4 \%$ \\
\hline
\end{tabular}

Table 5 Complication after TLIF operation 


\begin{tabular}{|ccc|c|c|}
\hline \multicolumn{5}{|c|}{ Complication $\square \mathrm{n} \square$} \\
\hline Parameter & $\begin{array}{c}\text { Observation } \\
\text { group }\end{array}$ & Control group 1 & Control group 2 & Control group 3 \\
\hline Infection & 0 & 0 & 0 & 1 \\
\hline Locf & 2 & 1 & 2 & 2 \\
\hline Ponv & 5 & 6 & 6 & 4 \\
\hline Total & $7 \square 15 \% \square$ & $7 \square 13 \% \square$ & $8 \square 17 \% \square$ & $6 \square 14 \% \square$ \\
\hline
\end{tabular}

Ponv $\llbracket$ post operation nausea and vomiting Locflleakage of cerebrospinal fluid The patient who infection in control group 3 is one of the patients in Locf

\section{Figures}

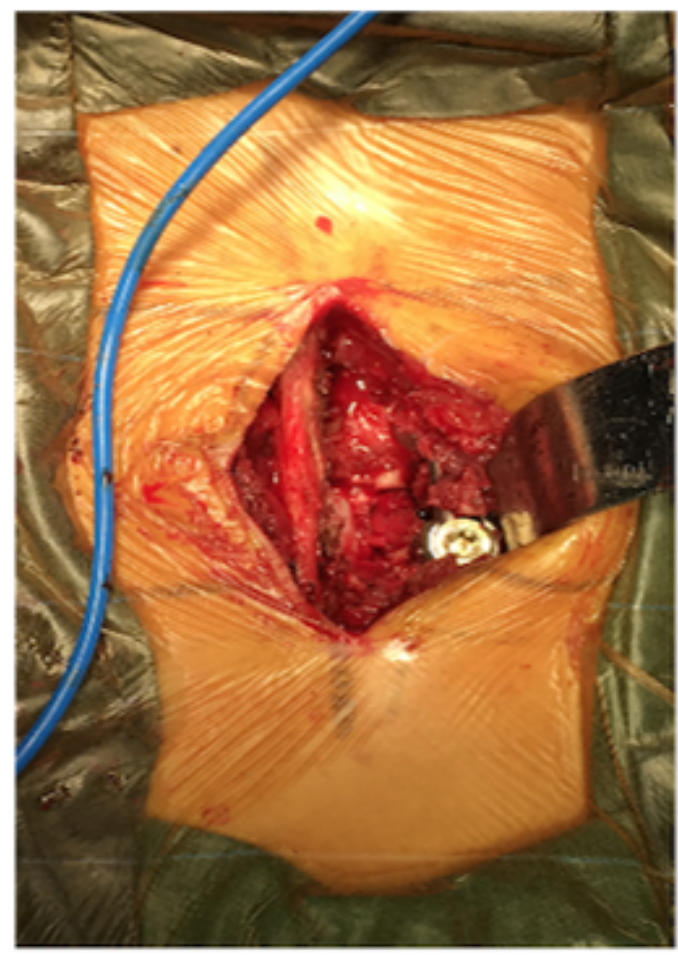

Figure 1

The Nerve root after decompression 


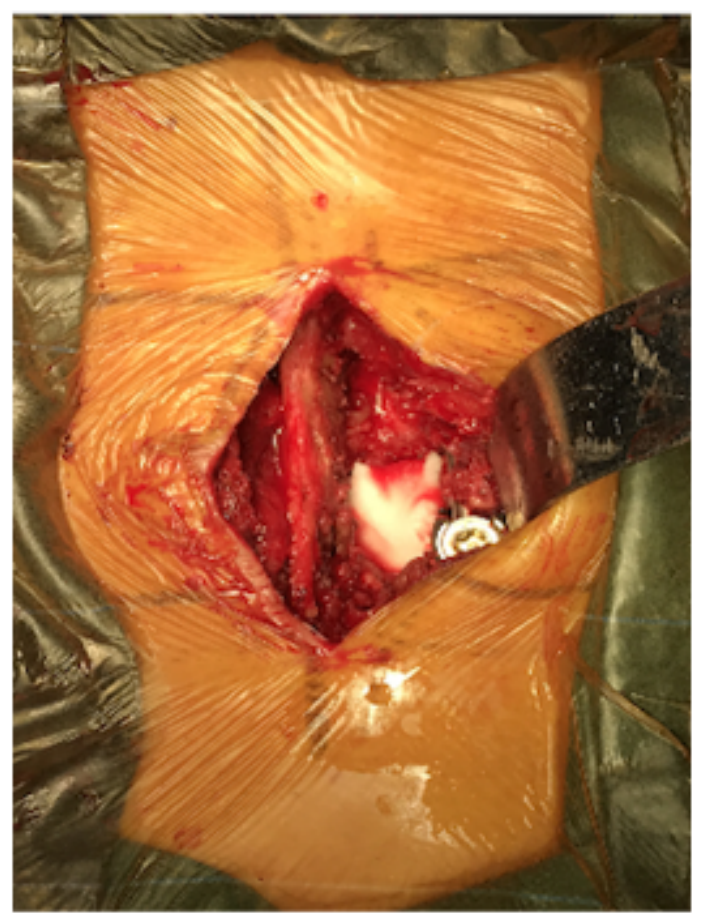

Figure 2

Put gelatin sponge on Nerve root

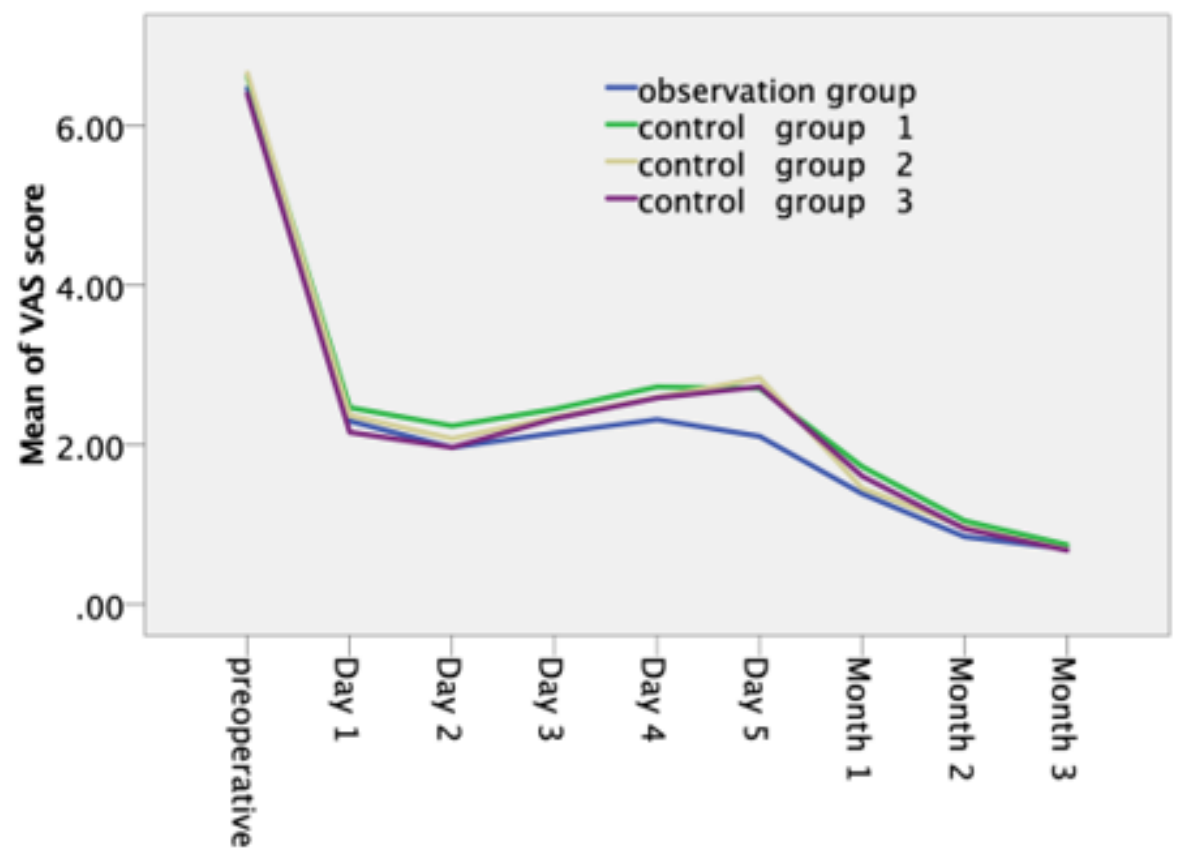

Figure 3

Mean of VAS score of leg pain from preoperative to postoperative 


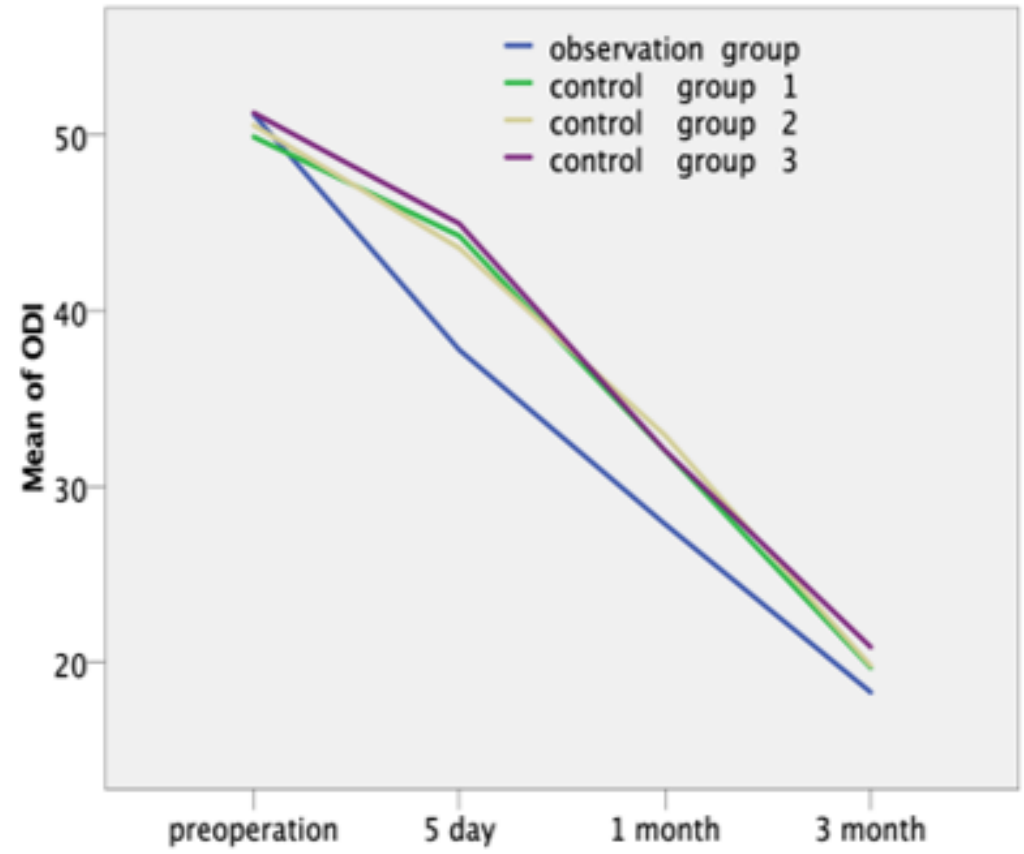

Figure 4

Mean of ODI from preoperative to postoperative 